\title{
Nutritional status and risk of contrast-associated acute kidney injury in elderly patients undergoing percutaneous coronary intervention
}

\author{
Xiaoqi Wei ${ }^{1}$. Hanchuan Chen ${ }^{2} \cdot$ Zhebin You $^{1}$. Jie Yang ${ }^{1} \cdot$ Haoming $\mathrm{He}^{2} \cdot$ Chen $\mathrm{He}^{2} \cdot$ Weiping Zheng ${ }^{1} \cdot$ Kaiyang Lin $^{2}$. \\ Feng Jiang ${ }^{1}$ (i)
}

Received: 10 January 2021 / Accepted: 2 April 2021 / Published online: 12 April 2021

(c) The Author(s) 2021

\begin{abstract}
Background This study aimed to investigate the connection between malnutrition evaluated by the Controlling Nutritional Status (CONUT) score and the risk of contrast-associated acute kidney injury (CA-AKI) in elderly patients who underwent percutaneous coronary intervention (PCI).

Methods A total of 1308 patients aged over 75 years undergoing PCI was included. Based on the CONUT score, patients were assigned to normal ( $(0-1)$, mild malnutrition (2-4), moderate-severe malnutrition group $(\geq 5)$. The primary outcome was CA-AKI (an absolute increase in $\geq 0.3 \mathrm{mg} / \mathrm{dL}$ or $\geq 50 \%$ relative serum creatinine increase $48 \mathrm{~h}$ after contrast medium exposure).

Results Overall, the incidence of CA-AKI in normal, mild, moderate-severe malnutrition group was $10.8 \%, 11.0 \%$, and $27.2 \%$, respectively $(p<0.01)$. Compared with moderate-severe malnutrition group, the normal group and the mild malnutrition group showed significant lower risk of CA-AKI in models adjusting for risk factors for CA-AKI and variables in univariate analysis (odds ratio $[\mathrm{OR}]=0.48,95 \%$ confidence interval $[\mathrm{CI}]: 0.26-0.89, p=0.02$; $\mathrm{OR}=0.46,95 \% \mathrm{CI}$ : $0.26-0.82$, $p=0.009$, respectively). Furthermore, the relationship were consistent across the subgroups classified by risk factors for CA-AKI except anemia. The risk of CA-AKI related with CONUT score was stronger in patients with anemia. (overall interaction $p$ by CONUT score $=0.012$ ).
\end{abstract}

Conclusion Moderate-severe malnutrition is associated with higher risk of CA-AKI in elderly patients undergoing PCI.

Keywords Malnutrition - Controlling Nutritional Status (CONUT) score · Contrast-associated acute kidney injury ·

Percutaneous coronary intervention · Elderly

Xiaoqi Wei and Hanchuan Chen contributed equally to this work

Kaiyang Lin

1ky7411@sina.com

$\triangle$ Feng Jiang

jiangfengconut@126.com

1 Department of Geriatric Medicine, Shengli Clinical Medical College of Fujian Medical University, Fujian Provincial Hospital, Fujian Provincial Institute of Clinical Geriatrics, Fujian Key Laboratory of Geriatrics, Fujian Provincial Center for Geriatrics, Fuzhou 350001, Fujian, China

2 Department of Cardiology, Shengli Clinical Medical College of Fujian Medical University, Fujian Provincial Hospital, Fujian Provincial Key Laboratory of Cardiovascular Disease, Fuzhou 350001, Fujian, China

\section{Introduction}

With the development of coronary angiography, more and more elderly patients with coronary heart disease receive percutaneous coronary intervention (PCI). However, elderly patients with coronary diseases who underwent percutaneous coronary intervention (PCI), were more likely to develop contrast-associated acute kidney injury (CA-AKI) than general population [1]. CA-AKI is a relatively common complication after intravascular contrast media administration, which significantly prolongs days for hospitalization, increases risk of mortality and morbidity [2]. Since therapeutic strategies for CA-AKI are limited, early screening of this high-risk population and implement preventive measurement are particularly important.

Malnutrition, which is high present in elderly patients $[3,4]$, is also a predisposing factor for AKI $[5,6]$. The 
Controlling Nutritional Status (CONUT) score, an objective and comprehensive tool for nutrition assessment, is calculated from the serum albumin value, the total cholesterol level, and the total lymphocyte count [7]. The prognostic value of the CONUT score has been proved in patients with coronary artery disease $[8,9]$. However, previous studies emphasized on the outcomes of mortality and adverse cardiovascular events. The role of the CONUT score in CAAKI, one of the adverse outcomes after PCI, has not been investigated in elderly patients undergoing PCI.

It has been demonstrated that each component of the CONUT score is related to kidney injury $[5,10,11]$. Therefore, we hypothesized that the CONUT score was associated with the incidence of CA-AKI in elderly patients undergoing PCI. We aim to evaluate the predictive value of the CONUT score for CA-AKI.

\section{Methods}

\section{Study population}

We conducted a retrospective cohort study at the Fujian Provincial Hospital, Fujian Cardiovascular Institute, between January 2012 and December 2018. A total of 1477 elderly patients aged $\geq 75$ years undergoing PCI were enrolled. The exclusion criteria were as follows: (1) died within $24 \mathrm{~h}$ after admission ( $n=3)$; (2) end-stage renal disease (estimated glomerular filtration rate $[\mathrm{eGFR}] \leq 15 \mathrm{~mL} / \mathrm{min} / 1.73 \mathrm{~m}^{2} ; n=5$ ); (3) history of radio-contrast agents $48 \mathrm{~h}$ prior to procedure or $72 \mathrm{~h}$ post-procedure $(n=3)$; (4) lack of data on pre-procedural or post-procedural serum creatinine ( $\mathrm{SCr}$ ) levels $(n=73)$; (5) lack of data of serum albumin level, the total cholesterol level, or the total lymphocyte count $(n=73)$; (6) history of malignant tumor with expectation of life less than 1 year $(n=9)$. Consequently, 1308 patients were eligible in the study (Fig. 1).

\section{Protocol}

Data collected included basic characteristics [e.g. age, hypertension, diabetes mellitus (DM)] and procedure related data (e.g. number of diseased vessels). The serum albumin level, total cholesterol level, and the lymphocyte count was measured for each patient at admission. SCr was measured at admission and daily for 3 days after contrast exposure. We also measured white blood cell (WBC) count, hemoglobin (HGB), hematocrit (HCT) and other standard clinical parameters on the morning of the first or second day after admission. The eGFR was calculated using the modified modification of diet in renal disease equation [12]: eGFR = $175 \times(\mathrm{SCr} / 88.4)^{-1.554} \times \mathrm{age}^{-0.203} \times 0.742$ (if female) $\times 1.212$

Fig. 1 Study population

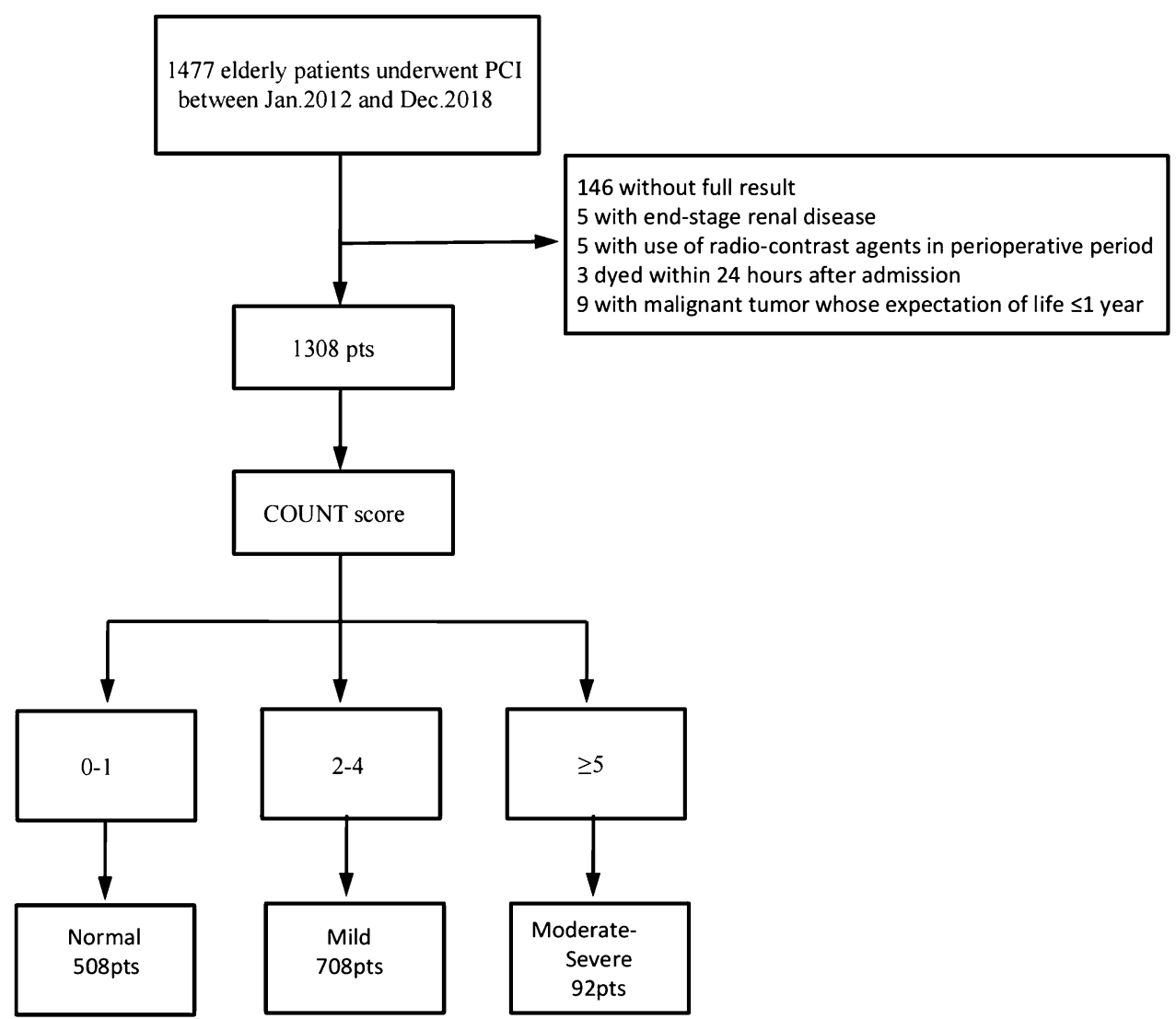


(if black). The use of medication was determined by the cardiologists according to clinical protocols based on guidelines. Left ventricular ejection fraction (LVEF) was evaluated using echo-cardiography during hospitalization. PCI was performed by experienced interventional cardiologists. All patients received nonionic contrast media (either Iopamiron or ultravist, both $370 \mathrm{mg} / \mathrm{mL}$ ). In addition, all patients received $0.9 \%$ normal saline (NS) at a rate of $1 \mathrm{~mL} / \mathrm{kg} / \mathrm{h}$ for $12 \mathrm{~h}$ during perioperative period(or $0.5 \mathrm{~mL} / \mathrm{kg} / \mathrm{h}$ for $12 \mathrm{~h}$ if patients had overt heart failure) [13]. The protocol met the requirements of the Declaration of Helsinki and was approved by the ethics committee of the Fujian Provincial Hospital, China (ethics approval number: K2012-01-011).

\section{Nutrition status evaluation}

Nutritional status was evaluated based on the CONUT score, which includes values of serum albumin, total cholesterol level, and total lymphocyte count, with a score ranging from 0 to 12 (Table S1) [7]. Higher scores indicates a worse nutritional status. Based on the CONUT score, patients were divided into normal (0-1), mild malnutrition (2-4), moderate-severe $(\geq 5)$ malnutrition groups. The classification of nutritional status has also been applied in other studies [14, $15]$.

\section{Definitions and end points}

The primary end point was the development of CA-AKI, defined as an absolute increase in $\geq 0.3 \mathrm{mg} / \mathrm{dL}$ or $\geq 50 \%$ from the baseline $\mathrm{SCr}$ levels within $48 \mathrm{~h}$ after exposure to contrast media $(\mathrm{CM})[16,17]$. Anemia was defined as $\mathrm{HCT}<0.39$ (for males) or, $<0.36$ (for females). The diagnosis of myocardial infarction (MI) is detection of cardiac troponin I (cTnI) above the 99th percentile upper reference limit (URL) and with one of the following: symptoms of ischaemia; new or presumed new significant ST-segment-T wave (ST-T) changes or new left bundle branch block (LBBB); development of pathological Q waves in the ECG; identification of an intracoronary thrombus by angiography [18].

\section{Statistical analyses}

All statistical analyses were performed using $\mathrm{R}$ version 4.0.2. The baseline characteristics were compared among three groups divided by CONUT score. Normally distributed continuous variables were expressed as mean \pm standard deviation (SD). The Student's $t$-test, Wilcoxon rank sum test was performed to determine the differences between CA-AKI and Non CA-AKI groups. And one way-analysis of variance was performed to determine the differences between groups classified by CONUT score. The categorical variables were represented as percentages and analyzed using chi-square test or Fisher's exact test.

After testing for proportional odds assumptions, multivariate logistic analysis was used to examine the association of CONUT score $0-1$ and CONUT score $2-4$ (vs. CONUT score $\geq 5$ ) with CA-AKI in models adjusted as follows: model 1 adjusted for traditional risk factors for CA-AKI (age, anemia, DM, contrast media(CM) volume $>200 \mathrm{ml}$, eGFR $<60 \mathrm{~mL} / \mathrm{min} / 1.73 \mathrm{~m}^{2}$ ); and model 2 adjusted for variables in model 1 plus the variables with $p$ value $<0.05$ in the univariate statistical results including $\mathrm{AF}$, emergency PCI, MI, perioperative hypotension, WBC, blood glucose, hyperuricemia (HUA). Interactions between the primary end point and prespecified subgroups stratified by several CAAKI risk factors were assessed using a likelihood ratio test for interaction. The $p$ values for interaction were calculated in each subgroup. A 2 -sided $p$ value $<0.05$ was considered as statistically significant.

\section{Result}

\section{Baseline characteristics}

A total of 1308 elderly patients were included in this study. Baseline characteristics are listed in Table 1. By CONUT calculations, 508 (38.8\%) patients were not malnourished, $708(54.1 \%)$ patients had mild malnutrition, $92(7.0 \%)$ patients had moderate-severe malnutrition, respectively. Patients with moderate-severe malnutrition were more likely to have MI, emergency PCI, anemia, hyperuricemia. They also had lower level of WBC, lymphocyte count, serum albumin, HCT, total cholesterol, but higher level of glucose, higher percentage of eGFR $<60 \mathrm{~mL} / \mathrm{min} / 1.73 \mathrm{~m}^{2}$ (all $p<0.05$ ).

And baseline characteristics between CA-AKI group and non CA-AKI group are presented in Table 2. Based on the CONUT score, 25 (15.8\%) patients in CA-AKI group and 67 (5.8\%) patients in non CA-AKI group had moderatesevere malnutrition, respectively. Patients who developed CA-AKI were more likely to have MI, emergency PCI, DM, AF, HUA, as well as higher level of WBC, blood glucose, lower level of serum albumin and lymphocyte count. More patients in the CA-AKI group were more likely to be treated with contrast volume of $\geq 200 \mathrm{~mL}$ (all $p<0.05$ ).

\section{Risk factors of CA-AKI}

The incidence of CA-AKI was $10.83 \%, 11.02 \%$, $27.17 \%$, from the group of CONUT $0-1$ to the group of CONUT $\geq 5$, respectively $(p<0.001)$ (Table 3$)$. After adjusting for traditional risk factors for CA-AKI, such as age, anemia, DM, CM $>200 \mathrm{ml}$, eGFR $<60 \mathrm{~mL} / \mathrm{min} / 1.73$ 
Table 1 Baseline characteristics of patients in different groups classified by CONUT score

\begin{tabular}{|c|c|c|c|c|}
\hline Variables & $\begin{array}{l}\text { Normal } \\
\text { (CONUT 0-1) } \\
N=508\end{array}$ & $\begin{array}{l}\text { Mild } \\
\text { (CONUT 2-4) } \\
N=708\end{array}$ & $\begin{array}{l}\text { Moderate-severe } \\
(\text { CONUT } \geq 5) \\
N=92\end{array}$ & $p$ Value \\
\hline \multicolumn{5}{|l|}{ Demographics } \\
\hline Age, years & 78.893 .34 & 79.093 .55 & 80.664 .48 & $<0.001$ \\
\hline Sex, female, $n(\%)$ & $194(38.19 \%)$ & $152(21.47 \%)$ & $20(21.74 \%)$ & $<0.001$ \\
\hline Systolic blood pressure, $\mathrm{mmHg}$ & 138.2423 .40 & 134.4422 .05 & 127.3020 .62 & $<0.001$ \\
\hline Diastolic blood pressure, $\mathrm{mmHg}$ & 72.2712 .43 & 71.5523 .58 & 67.9412 .71 & 0.155 \\
\hline Hypertension, $n(\%)$ & $377(74.2 \%)$ & $552(78.0 \%)$ & $74(80.4 \%)$ & 0.211 \\
\hline Diabetes, $n(\%)$ & $175(34.5 \%)$ & $260(36.7 \%)$ & $37(40.2 \%)$ & 0.498 \\
\hline Smoker, $n(\%)$ & $140(30.2 \%)$ & $229(36.2 \%)$ & $27(32.9 \%)$ & 0.114 \\
\hline Atrial fibrillation, $n(\%)$ & $62(12.2 \%)$ & $102(14.4 \%)$ & $12(13.0 \%)$ & 0.536 \\
\hline Emergency PCI, $n(\%)$ & $68(13.4 \%)$ & $109(15.4 \%)$ & $25(27.2 \%)$ & 0.003 \\
\hline Myocardial infarction, $n(\%)$ & $191(37.6 \%)$ & $304(42.9 \%)$ & $67(72.8 \%)$ & $<0.001$ \\
\hline Perioperative hypotension, $n(\%)$ & $45(8.9 \%)$ & $76(10.7 \%)$ & $18(19.6 \%)$ & 0.009 \\
\hline \multicolumn{5}{|l|}{ Laboratory measurements } \\
\hline Serum creatinine, $\mu \mathrm{mol} / \mathrm{L}$ & 84.4628 .86 & 89.6930 .60 & 108.0985 .05 & $<0.001$ \\
\hline $\mathrm{WBC}, \times 10^{9} / \mathrm{L}$ & 7.742 .44 & 7.392 .66 & 7.783 .45 & 0.049 \\
\hline lymphocyte, $\times 10^{9} / \mathrm{L}$ & 2.040 .58 & 1.510 .58 & 1.020 .40 & $<0.001$ \\
\hline $\mathrm{HGB}, \mathrm{g} / \mathrm{L}$ & 132.1514 .92 & 129.3416 .13 & 117.0720 .98 & $<0.001$ \\
\hline HCT & 0.390 .04 & 0.380 .04 & 0.350 .06 & $<0.001$ \\
\hline ALB, g/L & 40.733 .33 & 39.464 .04 & 33.274 .36 & $<0.001$ \\
\hline Cholesterol, mg/dL & 189.8839 .51 & 43.7032 .91 & 121.9727 .93 & $<0.001$ \\
\hline Glucose, $\mathrm{mmol} / \mathrm{L}$ & 7.122 .97 & 7.233 .08 & 9.024 .82 & $<0.001$ \\
\hline Uric acid, $\mu \mathrm{mol} / \mathrm{L}$ & 380.68102 .36 & 377.61108 .65 & 354.82121 .18 & 0.103 \\
\hline eGFR, $\mathrm{mL} / \mathrm{min} / 1.73 \mathrm{~m}^{2}$ & 80.3724 .70 & 78.9325 .36 & 70.1725 .31 & 0.002 \\
\hline $\mathrm{eGFR}<60 \mathrm{~mL} / \mathrm{min} / 1.73 \mathrm{~m}^{2}, n(\%)$ & $100(19.7 \%)$ & $156(22.0 \%)$ & $31(33.7 \%)$ & 0.011 \\
\hline Urine PH & 6.270 .71 & 6.290 .74 & 6.250 .72 & 0.885 \\
\hline Anemia, $n(\%)$ & $181(35.6 \%)$ & $331(46.8 \%)$ & $65(70.7 \%)$ & $<0.001$ \\
\hline Hyperuricemia, $n(\%)$ & $203(40.0 \%)$ & $243(34.3 \%)$ & $26(28.3 \%)$ & 0.035 \\
\hline \multicolumn{5}{|l|}{ Medical therapy during hospitalization } \\
\hline Statin, $n(\%)$ & $498(98.0 \%)$ & $699(98.7 \%)$ & $91(98.9 \%)$ & 0.637 \\
\hline ACEI/ARB, $n(\%)$ & $405(79.7 \%)$ & $586(82.8 \%)$ & $71(77.2 \%)$ & 0.242 \\
\hline Antiplatelet, $n(\%)$ & $498(98.0 \%)$ & $695(98.2 \%)$ & $89(96.7 \%)$ & 0.598 \\
\hline Metformin, $n(\%)$ & $73(14.4 \%)$ & $87(12.3 \%)$ & $12(11.0 \%)$ & 0.539 \\
\hline \multicolumn{5}{|l|}{ Procedure characteristic } \\
\hline Multi-vessel coronary artery disease, $n(\%)$ & $403(79.3 \%)$ & $569(80.4 \%)$ & $78(84.8 \%)$ & 0.479 \\
\hline Number of diseased vessels, $n(\%)$ & $2.32 \pm 0.82$ & $2.38 \pm 0.82$ & $2.45 \pm 0.75$ & 0.254 \\
\hline Number of stents, $n(\%)$ & $1.59 \pm 0.75$ & $1.68 \pm 0.80$ & $1.65 \pm 0.74$ & 0.160 \\
\hline Iso-osmolar contrast media use, $n(\%)$ & $172(33.9 \%)$ & $267(37.7 \%)$ & $34(37.0 \%)$ & 0.381 \\
\hline Volume of contrast media, mL & 177.0752 .39 & 177.7057 .76 & 179.7854 .41 & 0.909 \\
\hline Contrast volume $>200 \mathrm{~mL}, n(\%)$ & $88(17.3 \%)$ & $128(18.1 \%)$ & $19(20.7 \%)$ & 0.741 \\
\hline
\end{tabular}

$W B C$ white blood cell, $H G B$ hemoglobin, $H C T$ hematocrit, $A L B$ albumin, $e G F R$ estimated glomerular filtration rate, $P C I$ percutaneous coronary intervention, $A C E I$ angiotensin-converting enzyme inhibitor, $A R B$ angiotensin receptor blocker

$\mathrm{m}^{2}$, multiple logistic regression analysis confirmed that the group of CONUT score $0-1$ and the group of CONUT score 2-4 were associated with a lower risk of CA-AKI after PCI, compared with the group of CONUT score $\geq 5$ (odds ratio[OR] $0.40,95 \%$ confidence interval
[CI] 0.23-0.72, $p=0.002$; OR $0.40,95 \%$ CI $0.23-0.70$, $p=0.001$ ) (Table 3). After adjusting for variables in model 1 plus the other variables including AF, emergency PCI, MI, perioperative hypotension, WBC, blood glucose, hyperuricemia(HUA), the group of CONUT score $0-1$ 
Table 2 Baseline characteristics between non CA-AKI group and CA-AKI group

\begin{tabular}{|c|c|c|c|}
\hline & $\begin{array}{l}\text { Non CA-AKI } \\
N=1150\end{array}$ & $\begin{array}{l}\text { CA-AKI } \\
N=158\end{array}$ & $p$ Value \\
\hline \multicolumn{4}{|l|}{ Demographics } \\
\hline Age, years & 79.053 .49 & 79.704 .06 & 0.121 \\
\hline Sex, female, $n(\%)$ & $311(27.0 \%)$ & $55(34.8 \%)$ & 0.052 \\
\hline Systolic blood pressure, $\mathrm{mmHg}$ & 135.9622 .21 & 131.6425 .47 & 0.078 \\
\hline Diastolic blood pressure, $\mathrm{mmHg}$ & 71.6619 .93 & 71.0313 .88 & 0.723 \\
\hline Hypertension, $n(\%)$ & $877(76.3 \%)$ & $126(79.8 \%)$ & 0.384 \\
\hline Emergency PCI, $n(\%)$ & $153(13.3 \%)$ & $49(31.0 \%)$ & $<0.001$ \\
\hline Diabetes, $n(\%)$ & $590(51.3 \%)$ & $109(69.0 \%)$ & $<0.001$ \\
\hline Atrial fibrillation, $n(\%)$ & $142(12.4 \%)$ & $34(21.5 \%)$ & 0.002 \\
\hline Smoker, $n(\%)$ & $352(34.0 \%)$ & $44(30.4 \%)$ & 0.430 \\
\hline MI, $n(\%)$ & $455(39.6 \%)$ & $107(67.7 \%)$ & $<0.001$ \\
\hline Perioperative hypotension, $n(\%)$ & $96(8.3 \%)$ & $43(27.2 \%)$ & $<0.001$ \\
\hline \multicolumn{4}{|l|}{ Medical therapy during hospitalization } \\
\hline Statin, $n(\%)$ & $1132(98.4 \%)$ & $156(98.7 \%)$ & 1.000 \\
\hline Antiplatelet agents, $n(\%)$ & $1129(98.3 \%)$ & $153(96.8 \%)$ & 0.231 \\
\hline $\mathrm{ACEI} / \mathrm{ARB}, n(\%)$ & $931(81.0 \%)$ & $123(77.9 \%)$ & 0.413 \\
\hline Metformin, $n(\%)$ & $150(13.0 \%)$ & $21(13.3 \%)$ & 1.000 \\
\hline \multicolumn{4}{|l|}{ Laboratory measurements } \\
\hline Serum creatinine, $\mu \mathrm{mol} / \mathrm{L}$ & 88.0929 .88 & 95.2769 .28 & 0.597 \\
\hline $\mathrm{WBC}, \times 10^{9} / \mathrm{L}$ & 7.392 .48 & 8.723 .42 & $<0.001$ \\
\hline Lymphocyte, $\times 10^{9} / \mathrm{L}$ & 1.700 .64 & 1.540 .69 & 0.005 \\
\hline $\mathrm{HGB}, \mathrm{g} / \mathrm{L}$ & 129.9616 .06 & 126.7119 .03 & 0.099 \\
\hline $\mathrm{HCT}$ & 0.380 .05 & 0.370 .05 & 0.073 \\
\hline $\mathrm{ALB}, \mathrm{g} / \mathrm{L}$ & 39.784 .15 & 37.574 .21 & $<0.001$ \\
\hline Cholesterol, mg/dL & 159.8543 .50 & 161.9838 .06 & 0.187 \\
\hline Glucose, $\mathrm{mmol} / \mathrm{L}$ & 7.173 .07 & 8.384 .01 & $<0.001$ \\
\hline $\mathrm{eGFR}, \mathrm{mL} / \mathrm{min} / 1.73 \mathrm{~m}^{2}$ & 78.9323 .75 & 78.4734 .06 & 0.247 \\
\hline $\mathrm{eGFR}<60 \mathrm{~mL} / \mathrm{min} / 1.73 \mathrm{~m}^{2}, n(\%)$ & $242(21.0 \%)$ & $45(28.5 \%)$ & 0.044 \\
\hline Urine PH & 6.290 .72 & 6.220 .80 & 0.090 \\
\hline CONUT score & & & $<0.001$ \\
\hline $0-1, n(\%)$ & $453(39.4 \%)$ & $55(34.8 \%)$ & \\
\hline $2-4, n(\%)$ & $630(54.8 \%)$ & $78(49.4 \%)$ & \\
\hline$\geq 5, n(\%)$ & $67(5.8 \%)$ & $25(15.8 \%)$ & \\
\hline Anemia, $n(\%)$ & $267(23.2 \%)$ & $48(30.4 \%)$ & 0.061 \\
\hline Hyperuricemia, $n(\%)$ & $395(34.4 \%)$ & $77(48.7 \%)$ & 0.001 \\
\hline \multicolumn{4}{|l|}{ Procedure characteristic } \\
\hline Contrast volume, $\mathrm{mL}$ & 176.1455 .91 & 188.2351 .01 & 0.006 \\
\hline Contrast volume $\geq 200 \mathrm{~mL}, n(\%)$ & $190(16.5 \%)$ & $45(28.5 \%)$ & 0.003 \\
\hline Iso-osmolar contrast media use, $n(\%)$ & $416(36.2 \%)$ & $57(36.1 \%)$ & 1.000 \\
\hline Number of stents, $n(\%)$ & 1.650 .78 & 1.580 .73 & 0.388 \\
\hline Multi-vessel coronary artery disease, $n(\%)$ & $915(79.57 \%)$ & $135(85.44 \%)$ & 0.102 \\
\hline
\end{tabular}

$M I$ myocardial infarction, $P C I$ percutaneous coronary intervention, $A C E I$ angiotensin-converting enzyme inhibitor, $A R B$ angiotensin receptor blocker, $e G F R$ estimated glomerular filtration rate, $A L B$ albumin, $W B C$ white blood cell, $H G B$ hemoglobin, $H C T$ hematocrit and the group of CONUT score 2-4 remained significant lower risk of CA-AKI in elderly patients after PCI, compared with the group of CONUT score $\geq 5$ (OR 0.48 , 95\% CI $0.26-0.89, p=0.02$; OR $0.46,95 \%$ CI $0.26-0.82$, $p=0.009$ ) (Table 3). In other words, CONUT score $\geq 5$ was independently associated with a higher risk of CAAKI, compared with CONUT score $0-1$ and CONUT score $2-4$.

The effects of the CONUT score on the rate of CA-AKI were consistent across the prespecified subgroups (HUA, 
Table 3 Associations between CONUT score and CA-AKI

\begin{tabular}{llllllll}
\hline & Participants, $n$ & Events, $n$ & Rate, $\%$ & $\begin{array}{l}\text { Model 1* } \\
\text { OR (95\%CI) }\end{array}$ & $p$ Value & $\begin{array}{l}\text { Model 2 } \\
\text { OR (95\%CI) }\end{array}$ \\
\hline CONUT score 0-1 & 508 & 55 & 10.83 & $0.40(0.23-0.72)$ & 0.002 & $0.48(0.26-0.89)$ & 0.02 \\
CONUT score 2-4 & 708 & 78 & 11.02 & $0.40(0.23-0.70)$ & 0.001 & $0.46(0.26-0.82)$ & 0.009 \\
CONUT score 25 & 92 & 25 & 27.17 & Reference & - & Reference & - \\
\hline
\end{tabular}

* Model 1 adjusted for age, anemia, diabetes, contrast media volume $>200 \mathrm{ml}$, eGFR $<60 \mathrm{ml} /\left(\mathrm{min} \cdot 1.73 \mathrm{~m}^{2}\right)$. ${ }^{\dagger}$ Model 2 adjusted for variables in model 1 plus atrial fibrillation, emergency PCI, MI, WBC, glucose, hyperuricemia, perioperative hypotension. $C I$ confidence interval, $O R$ odds ratio

DM, CM, eGFR, AF, emergency PCI, MI) (Fig. 2). However, there was an modification by anemia: the risk of CAAKI related with CONUT score was stronger in patients with anemia than in those without anemia (overall interaction $p$ by CONUT score $=0.012$ ) (Figs. 2, 3).

\section{Discussion}

To our knowledge, this is the first study to demonstrate the relationship between the objective nutrition scoring tool "CONUT" and the incidence of CA-AKI in elderly patients undergoing PCI. Our results show that the CONUT score $\geq 5$, recognized as moderate or severe malnutrition, is associated with an increased risk of CA-AKI in elderly patients undergoing PCI. Moreover, the association is stronger in elderly patients with anemia.

In our study, the incidence of CA-AKI in elderly patients undergoing PCI was up to $12.1 \%$, which was almost consistent with the data available in a meta-analysis [19]. Age over 75 years was widely recognized as an independent risk factor of CA-AKI in patients after PCI [1]. It's well-known that comorbidities are high present in the admitted elderly patients [20], such as the age-related decrease in kidney function [21] and anemia [22], which were identified as risk factors of CA-AKI [1,23]. Due to multiple chronic diseases, the elderly patients often take multiple oral drugs, which may worsen kidney function. Moreover, as poor vascular and heart condition, adequate hydration is difficult to achieve and thus nephrotoxic contrast cannot be discharged as soon as possible. Therefore, the elderly is more vulnerable. It's important to effectively further identify elderly patients at higher risk for CA-AKI and implement precise prevention.

In addition to the above factors, malnutrition is high present in elderly patients [3], which is also associated with the development of kidney injury [24]. However, the best tool to identify patients at high risk of malnutrition is still in dispute. Single nutrition indicators are often affected by many factors [25]. Subjective comprehensive nutritional scoring systems like Subjective Global Assessment (SGA), are sophisticated, which need the assistance of specialized nutritionists. But there are some objective comprehensive nutritional scoring systems such as the CONUT score, Prognostic Nutritional Index (PNI), which only require simple blood biomarkers and make it convenient to apply in clinic. The CONUT score is calculated from the serum albumin value, the total cholesterol level, and the total lymphocyte count [7], which only included one more indicator than PNI. The CONUT score has been used for assessing the prognosis of heart failure [15], acute ischemic stroke [26], and a variety of malignant tumors [27, 28]. Recently, the prognostic value of the CONUT score has been validated and shown better than PNI in patients with coronary artery disease. In an observational, retrospective study of 3118 cohort, patients undergoing PCI were divided into four groups by their CONUT score (0-1 vs. $2-4$ vs. $5-8$ vs. $9-12)$. It revealed that patients with higher CONUT scores had higher rates of major adverse cardiac events (hazard ratio [HR]: 1.14; 95\% CI: $1.07-1.22, p<0.05)$ [8]. Basta et al. evaluated the CONUT and PNI score in 945 patients with ST-elevation myocardial infarction undergoing PCI and found that patients with severe CONUT but not patients with severe PNI index had the highest rate for all-cause death, with a log-rank of $p<0.001$ [9]. Roubín et al. made a similar conclusion that the CONUT score has a higher sensitivity than the PNI for all-cause death and major adverse cardiovascular events (MACEs) in patients with acute coronary syndrome [29]. Therefore, it was appropriate to apply the CONUT score in our study. However, these studies emphasized on the outcomes such as mortality and adverse cardiovascular events in patients after PCI. The role of the CONUT score in CA-AKI, which is one of the adverse outcomes of PCI, has not been investigated in elderly patients.

Our study first revealed that elderly patients with moderate-severe malnutrition (CONUT score $\geq 5$ ) had higher risk of CA-AKI. Even after adjusting for multiple risk factors, moderate-severe malnutrition is also significantly associated with the development of CA-AKI. Our subgroup analysis also confirms the relationship between the CONUT score and the risk of CA-AKI, although there is an modification by anemia. This could probably be explained by the added risk of anemia to CA-AKI. It was recognized that anemia on admission is associated with an increased risk of CA-AKI [1]. The possible explanation may be the anemia-induced 


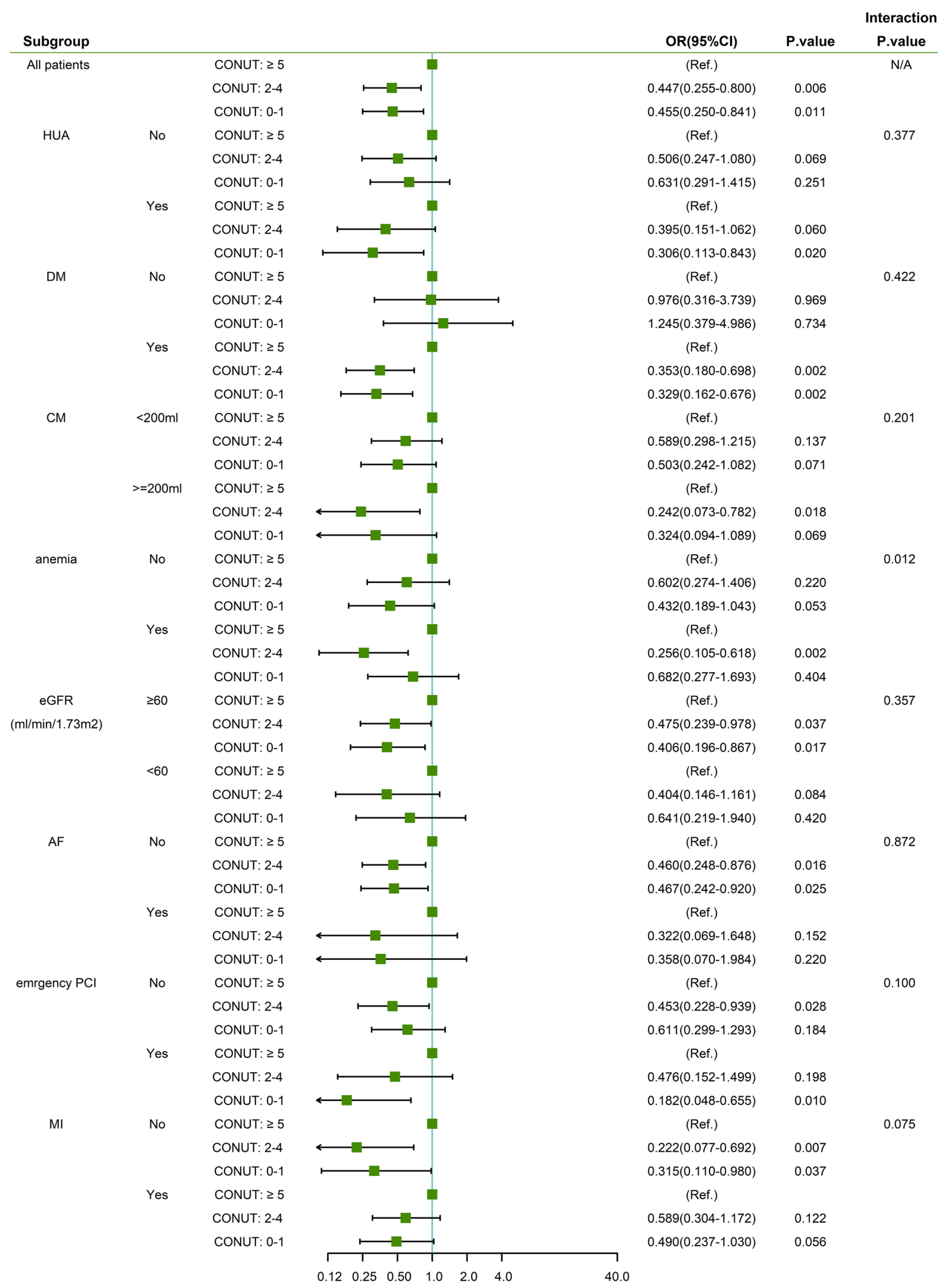

Fig. 2 Subgroup analysis of the effect of CONUT score on CA-AKI incidence in the matched cohort 
Fig. 3 Incidence rate of CA$\mathrm{AKI}$ in patients with various combination of CONUT score and anemia

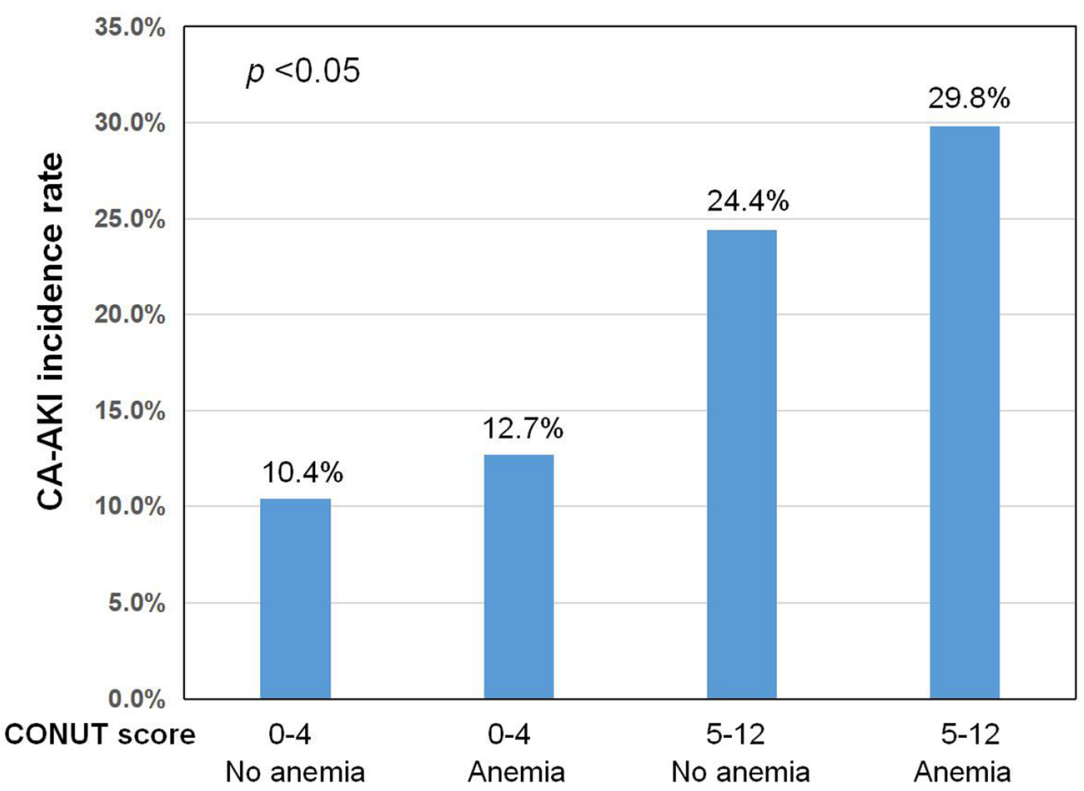

\begin{tabular}{|l|c|c|c|c|}
\hline Cl-AKI & 99 & 34 & 11 & 14 \\
\hline No Cl-AKI & 849 & 234 & 34 & 33 \\
\hline Total & 948 & 268 & 45 & 47 \\
\hline $\begin{array}{l}\text { Incidence } \\
\text { rate(\%) }\end{array}$ & 10.4 & 12.7 & 24.4 & 29.8 \\
\hline
\end{tabular}

aggravation of renal ischemia and hypoxic injury to the outer medulla in the kidney [30]. Combined with malnutrition, the capacity of scavenging oxygen free radical is reduced, which may aggravate cell toxicity and further impair the kidney function [31].

The mechanism of the connection between the CONUT score and CA-AKI has not been fully investigated. However, each component of CONUT score has been demonstrated the relationship with kidney injury. First, serum albumin levels, which are twice the weight of other indicators in CONUT score, can predict the risk of CA-AKI. A meta-analysis including about 68,000 subjects confirmed that lower serum albumin is an independent predictor both of AKI and death after AKI in patients undergoing cardiac surgery or acute coronary interventions [6]. Furthermore, a retrospective study found that in patients treated with PCI, the serum albumin level was significantly lower in the CA-AKI group $(3.52+0.40$ vs. $3.94+0.39 \mathrm{mg} / \mathrm{dL}, p<00.001)$ and it was an independent predictor of CA-AKI[5]. Second, although hypercholesterolemia is a well-established risk factor for cardiovascular disease in the general population [32], the correlation between low serum cholesterol and adverse outcomes has been reported for patients with renal failure. Obialo et al. evaluated a 3-year retrospective study of patients with acute kidney injury and demonstrated that survival was higher among patients with cholesterol $>150 \mathrm{mg} / \mathrm{dL}$ than those whose levels were $<$ or $=150 \mathrm{mg} / \mathrm{dL}(p<0.001)$ [10]. Another study also showed that lower total cholesterol (TC) levels over time were significantly associated with worse survival (HR 1.66, 95\% CI 1.11-2.47) in patients with chronic kidney diseases who underwent peritoneal dialysis [33]. Finally, lymphocyte count may represent a marker of the inflammation response [34], which is a significant and crucial factor in the pathogenesis of CA-AKI [35]. A low relative lymphocyte count was shown to be independently associated with worse prognosis in patients with CAD [36]. Additionally, several studies have shown that higher ratio of neutrophil/lymphocyte is related to a greater risk of CA-AKI in patients who underwent PCI [11,37], suggesting that a low lymphocyte count may contribute to the development of CA-AKI. Thus, the CONUT score, reflecting not only the status of malnutrition but also the degree of inflammation, may be more appropriate for early detection of elderly patients with high risk of CA-AKI.

\section{Limitation}

First, this study was a single-center cohort with a relatively small sample size. A potential patient selection bias may be existed. Second, the CONUT score was evaluated only at the time of admission, and we did not assess the effect of the change of score during the observation period. Thirdly, 
elderly patients comprised major proportion of participants in our study. Therefore, our results may not be applied to younger patients. Furthermore, based on this study, it is unclear whether the stratified risk classes need an nutritional intervention.

\section{Conclusion}

We find that moderate-severe malnutrition is strongly associated with high risk of CA-AKI in elderly patients who underwent PCI. Moreover, the risk of CA-AKI related with CONUT score was stronger in patients with anemia. Further studies are required to determine whether nutrition support improves clinical outcomes in this population.

Supplementary Information The online version contains supplementary material available at https://doi.org/10.1007/s10157-021-02061-4.

Author contributions XW: methodology, validation, investigation, visualization, writing — original draft preparation; HC: conceptualization, validation, investigation; $\mathrm{ZY}$ : software, formal analysis, data curation; JY: investigation; $\mathrm{H}-\mathrm{MH}$ : investigation; $\mathrm{CH}$ : investigation; WZ: writing-review and editing, funding acquisition; KL: conceptualization, methodology, formal analysis, funding acquisition; FJ: project administration, supervision.

Funding This research was funded by a grant from the Joint Funds for the innovation of science and technology, Fujian province (Grant number: 2018Y9097), high-level hospital foster grants from Fujian Provincial Hospital, Fujian province, China (Grant number: 2020HSJJ05), Fujian provincial health technology project (Grant number: 2019ZQN-10), General project of Science and Technology, Fujian Province (Grant number: 2018J01247), young and middle-aged personnel training project of Fujian provincial health commission (Grant number: 2018-ZQN-10).

\section{Declarations}

Conflict of interest The authors declare that there are no conflict of interests.

Ethical approval All procedures performed in studies involving human participants were in accordance with the ethical standards of the institutional or national research committee and with the 1964 Helsinki Declaration and its later amendments or comparable ethical standards. Approval for this study was approved by the ethics committee of the Fujian Provincial Hospital, China (ethics approval number: K201201-011).

Informed consent Informed written consents were obtained from all patients after providing them a detailed written description of the potential benefits and risks associated with the study.

Open Access This article is licensed under a Creative Commons Attribution 4.0 International License, which permits use, sharing, adaptation, distribution and reproduction in any medium or format, as long as you give appropriate credit to the original author(s) and the source, provide a link to the Creative Commons licence, and indicate if changes were made. The images or other third party material in this article are included in the article's Creative Commons licence, unless indicated otherwise in a credit line to the material. If material is not included in the article's Creative Commons licence and your intended use is not permitted by statutory regulation or exceeds the permitted use, you will need to obtain permission directly from the copyright holder. To view a copy of this licence, visit http://creativecommons.org/licenses/by/4.0/.

\section{References}

1. Mehran R, Aymong ED, Nikolsky E, et al. A simple risk score for prediction of contrast-induced nephropathy after percutaneous coronary intervention: development and initial validation. $\mathbf{J}$ Am Coll Cardiol. 2004;44(7):1393-9.

2. McCullough PA, Choi JP, Feghali GA, et al. Contrast-induced acute kidney injury. J Am Coll Cardiol. 2016;68(13):1465-73.

3. Ljungqvist O, van Gossum A, Sanz ML, de Man F. The European fight against malnutrition. Clin Nutr. 2010;29(2):149-50.

4. Kaiser MJ, Bauer JM, Rämsch C, et al. Frequency of malnutrition in older adults: a multinational perspective using the mini nutritional assessment. J Am Geriatr Soc. 2010;58(9):1734-8.

5. Murat SN, Kurtul A, Yarlioglues M. Impact of serum albumin levels on contrast-induced acute kidney injury in patients with acute coronary syndromes treated with percutaneous coronary intervention. Angiology. 2015;66(8):732-7.

6. Wiedermann CJ, Wiedermann W, Joannidis M. Causal relationship between hypoalbuminemia and acute kidney injury. World J Nephrol. 2017;6(4):176-87.

7. Ignacio de Ulíbarri J, González-Madroño A, de Villar NG, et al. CONUT: a tool for controlling nutritional status. First validation in a hospital population. Nutr Hosp. 2005;20(1):38-45.

8. Chen SC, Yang YL, Wu CH, et al. Association between preoperative nutritional status and clinical outcomes of patients with coronary artery disease undergoing percutaneous coronary intervention. Nutrients. 2020;12(5):1295.

9. Basta G, Chatzianagnostou K, Paradossi U, et al. The prognostic impact of objective nutritional indices in elderly patients with ST-elevation myocardial infarction undergoing primary coronary intervention. Int J Cardiol. 2016;221:987-92.

10. Obialo CI, Okonofua EC, Nzerue MC, Tayade AS, Riley LJ. Role of hypoalbuminemia and hypocholesterolemia as copredictors of mortality in acute renal failure. Kidney Int. 1999;56(3):1058-63.

11. Yuan Y, Qiu H, Hu X, et al. Predictive value of inflammatory factors on contrast-induced acute kidney injury in patients who underwent an emergency percutaneous coronary intervention. Clin Cardiol. 2017;40(9):719-25.

12. Levey AS, Coresh J, Greene T, et al. Using standardized serum creatinine values in the modification of diet in renal disease study equation for estimating glomerular filtration rate. Ann Intern Med. 2006;145(4):247-54.

13. Isaka $\mathrm{Y}$, Hayashi $\mathrm{H}$, Aonuma $\mathrm{K}$, et al. Guideline on the use of iodinated contrast media in patients with kidney disease 2018 . Clin Exp Nephrol. 2020;24(1):1-44.

14. Naito H, Nezu T, Hosomi N, et al. Controlling nutritional status score for predicting 3-mo functional outcome in acute ischemic stroke. Nutrition. 2018;55-56:1-6.

15. Agra Bermejo RM, González Ferreiro R, Varela Román A, et al. Nutritional status is related to heart failure severity and hospital readmissions in acute heart failure. Int J Cardiol. 2017;230:108-14.

16. Miura T, Miyashita Y, Motoki H, et al. Efficacy and safety of percutaneous coronary intervention for elderly patients in the 
second-generation drug-eluting stent era: the SHINANO Registry. Angiology. 2017;68(8):688-97.

17. Davenport MS, Perazella MA, Yee J, et al. Use of intravenous iodinated contrast media in patients with kidney disease: consensus statements from the American College of Radiology and the National Kidney Foundation. Radiology. 2020;294(3):660-8.

18. Thygesen K, Alpert JS, Jaffe AS, et al. Third universal definition of myocardial infarction. Eur Heart J. 2012;33(20):2551-67.

19. Song W, Zhang T, Pu J, Shen L, He B. Incidence and risk of developing contrast-induced acute kidney injury following intravascular contrast administration in elderly patients. Clin Interv Aging. 2014;9:85-93.

20. Fabbian F, De Giorgi A, Maietti E, et al. A modified Elixhauser score for predicting in-hospital mortality in internal medicine admissions. Eur J Intern Med. 2017;40:37-42.

21. Glassock RJ, Rule AD. Aging and the kidneys: anatomy, physiology and consequences for defining chronic kidney disease. Nephron. 2016;134(1):25-9.

22. Eisenstaedt R, Penninx BW, Woodman RC. Anemia in the elderly: current understanding and emerging concepts. Blood Rev. 2006;20(4):213-26.

23. Chen $\mathrm{H}, \mathrm{He} \mathrm{C}$, You $\mathrm{Z}$, et al. Association between urine $\mathrm{pH}$ and risk of contrast-associated acute kidney injury among patients after emergency percutaneous coronary intervention: a V-shape relationship. Clin Exp Nephrol. 2021. https://doi.org/10.1007/ s10157-020-02015-2.

24. Li C, Xu L, Guan C, et al. Malnutrition screening and acute kidney injury in hospitalised patients: a retrospective study over a 5-year period from China. Br J Nutr. 2020;123(3):337-46.

25. Berbel MN, Pinto MP, Ponce D, Balbi AL. Nutritional aspects in acute kidney injury. Rev Assoc Med Bras (1992). 2011;57(5):600-6.

26. Cai $\mathrm{ZM}, \mathrm{Wu} \mathrm{YZ}$, Chen HM, et al. Being at risk of malnutrition predicts poor outcomes at 3 months in acute ischemic stroke patients. Eur J Clin Nutr. 2020;74(5):796-805.

27. Ahiko Y, Shida D, Horie T, et al. Controlling nutritional status (CONUT) score as a preoperative risk assessment index for older patients with colorectal cancer. BMC Cancer. 2019;19(1):946.

28. Hirahara N, Tajima Y, Fujii Y, et al. Controlling Nutritional Status (CONUT) as a prognostic immunonutritional biomarker for gastric cancer after curative gastrectomy: a propensity scorematched analysis. Surg Endosc. 2019;33(12):4143-52.

29. Raposeiras Roubín S, Abu Assi E, Cespón Fernandez M, et al. Prevalence and prognostic significance of malnutrition in patients with acute coronary syndrome. J Am Coll Cardiol. 2020;76(7):828-40.

30. Kim SJ, Salem MR, Joseph NJ, Madayag MA, Cavallino RP, Crystal GJ. Contrast media adversely affect oxyhemoglobin dissociation. Anesth Analg. 1990;71(1):73-6.

31. Taverna M, Marie AL, Mira JP, Guidet B. Specific antioxidant properties of human serum albumin. Ann Intensive Care. 2013;3(1):4.

32. Mach F, Baigent C, Catapano AL, et al. 2019 ESC/EAS Guidelines for the management of dyslipidaemias: lipid modification to reduce cardiovascular risk. Eur Heart J. 2020;41(1):111-188.

33. Park CH, Kang EW, Park JT, et al. Association of serum lipid levels over time with survival in incident peritoneal dialysis patients. J Clin Lipidol. 2017;11(4):945-954.e3.

34. Bergquist J, Tarkowski A, Ewing A, Ekman R. Catecholaminergic suppression of immunocompetent cells. Immunol Today. 1998;19(12):562-7.

35. Seeliger E, Sendeski M, Rihal CS, Persson PB. Contrast-induced kidney injury: mechanisms, risk factors, and prevention. Eur Heart J. 2012;33(16):2007-15.

36. Bian $\mathrm{C}, \mathrm{Wu} \mathrm{Y}$, Shi $\mathrm{Y}$, et al. Predictive value of the relative lymphocyte count in coronary heart disease. Heart Vessels. 2010;25(6):469-73.

37. Zorlu C, Koseoglu C. Comparison of the relationship between inflammatory markers and contrast-induced nephropathy in patients with acute coronary syndrome after coronary angiography. Angiology. 2020;71(3):249-55.

Publisher's Note Springer Nature remains neutral with regard to jurisdictional claims in published maps and institutional affiliations. 